\title{
INVESTIGACIONES
}

\section{Las teorías implícitas sobre la comprensión textual y las estrategias metacognitivas de estudiantes universitarios de primer año*}

\author{
Implicit theories of text comprehension and metacognitive strategies in first year \\ university students
}

\begin{abstract}
As teorias implícitas sobre a compreensão textual e as estratégias metacognitivas de estudantes universitários de primeiro ano
\end{abstract}

Margarita Makuc. ${ }^{a}$

a Universidad de Magallanes, Chile. Fono: 61-207197. Correo electrónico: margarita.makuc@umag.cl

\begin{abstract}
RESUMEN
En este artículo se presentan los resultados obtenidos por estudiantes universitarios de primer año en la aplicación del cuestionario de teorías implícitas sobre la comprensión textual y el cuestionario de metacomprensión. Las teorías implícitas sobre la comprensión textual corresponden a tres teorías: teoría lineal, interactiva y literaria, y respecto de tres componentes: noción de comprensión, de lector y de texto. Por su parte, el cuestionario de metacomprensión nos permite abordar las estrategias metacognitivas en la lectura comprensiva, en relación al texto, al lector y a la tarea. El objetivo es identificar la relación entre una determinada teoría y las estrategias del lector, las que a su vez determinan diversos niveles de experticia lectora. Tanto las teorías implícitas como el razonamiento metacognitivo, constituyen un tipo de conocimiento implícito que los lectores hábiles deben activar en la resolución de tareas de lectura.
\end{abstract}

Palabras clave: teorías implícitas sobre la comprensión textual, estrategias metacognitivas, experticia lectora.

\begin{abstract}
This article presents the results obtained by university freshmen in the application of the questionnaire based on implicit theories of text comprehension and the questionnaire of metacomprehension. The implicit theories of textual understanding correspond to three theories: linear, interactive and literary theory and with respect to three components: notion of comprehension, reader and text. Moreover, the questionnaire of metacomprehension allows us to approach the metacognitive strategies in reading comprehension, in relation to the text, to the reader, and to the task. The objective is to identify the relationship between a particular theory and the strategies applied by the reader, which in turn determine different levels of reading expertise. Both implicit theories and the metacognitive reasoning, constitute a kind of implicit knowledge which skilled readers should activate for the resolution of reading tasks.
\end{abstract}

Key words: implicit theories about the textual understanding, metacognitive strategies, expertise of the reader.

Artículo elaborado en el marco del Proyecto FONDECYT REGULAR ( $\left.\mathrm{N}^{\circ} 1120891\right)$ : "Las Teorías implícitas sobre la comprensión y las estrategias del lector: aspectos de una relación dinámica y significativa en el desarrollo de las competencias lectoras de estudiantes universitarios de primer año". Resultados correspondientes a la primera etapa del proyecto (año 2012). Dra. Margarita Makuc Sierralta (Investigadora Principal), Universidad de Magallanes. Dra. Elisa Larrañaga Rubio (Colaboradora Internacional), Universidad de Castilla La Mancha. 


\begin{abstract}
RESUMO
Apresentam-se os resultados obtidos por estudantes universitários de primeiro ano na aplicação do questionário de teorias implícitas sobre a compreensão textual e o questionário de metacompreensão. As teorias implícitas sobre a compreensão textual correspondem a três teorias: a linear, a interativa e a literária e envolvem três componentes: noção de compreensão, de leitor e de texto. Por sua vez, o questionário de metacompreensão permite-nos abordar as estratégias metacognitivas na leitura compreensiva, em relação ao texto, ao leitor e à tarefa. O objetivo é identificar a relação entre uma determinada teoria e as estratégias do leitor que, por sua vez, determinam diferentes níveis de experiência leitora. Tanto as teorias implícitas como o raciocínio metacognitivo constituem um tipo de conhecimento implícito que os leitores hábeis devem ativar na resolução de tarefas de leitura.
\end{abstract}

Palavras chave: teorias implícitas sobre a compreensão, estratégias textuais metacognitivas, experiência leitora.

\title{
1. INTRODUCCIÓN
}

Diversas investigaciones han permitido determinar que los lectores pueden agruparse en torno a determinadas teorías sobre la comprensión textual que guiarían y orientarían sus acciones (Makuc, 2012, 2011, 2009, 2008; Hernández, 2008; Peronard, Velázquez, Crespo y Viramonte, 2002; Peronard, Crespo y Velásquez, 2000; Schraw, 2000; Schraw \& Bruning, 1996). Los estudios en diversos niveles de escolaridad evidencian que los estudiantes evolucionan desde una concepción lineal hacia una concepción interactiva, al mismo tiempo que sus estrategias se complejizan progresivamente (Peronard, 2005, 2002). Este desarrollo de la concepción de la lectura y de las estrategias muestra que los estudiantes descubren que la lectura referente es un proceso interactivo que se debe entender dentro de un marco comunicativo. Como consecuencia, su metaconocimiento incluye la idea de que debe integrar su conocimiento previo acerca del mundo y del tema para lograr construir su propia interpretación coherente del texto.

Como podemos observar en los estudios citados, la relación entre teorías de la lectura y estrategias lectoras es un aspecto significativo que se manifiesta, además, en la experticia lectora de los estudiantes. Se ha observado en estudiantes universitarios del área humanística que aquellos que adhieren a una teoría lineal, aun cuando obtienen buenos resultados en preguntas literales (nivel local), son superados por aquellos estudiantes en los que predomina una teoría interactiva, los que además se destacan por obtener los mejores resultados en preguntas de tipo inferencial tanto en el nivel local como en el nivel global (Makuc, 2011, 2009).

En esta línea de investigación, nos parece central complementar los estudios sobre comprensión textual, focalizando nuestra atención en la relación entre las teorías implícitas sobre la comprensión y las estrategias metacognitivas de los estudiantes universitarios. Desde este enfoque, se plantea integrar las representaciones del lector, que en términos de teorías implícitas movilizan sus acciones, orientándolo respecto de cómo dirigir su atención y canalizar sus recursos metacognitivos hacia determinadas metas que, desde su comprensión, resultan adecuadas y pertinentes.

En este contexto, la identificación tanto de las teorías implícitas como de las estrategias metacognitivas en el ámbito de la comprensión textual es considerada como parte de una secuencia, que se enmarca en el supuesto de que la explicitación del conocimiento del lector es un proceso gradual, y de que en todo conocimiento hay componentes implícitos y explícitos. En esta dirección, tanto el reconocimiento de afirmaciones que describen la 
comprensión textual en sus componentes centrales (noción de comprensión, de lector y de texto) como el reconocimiento de estrategias metacognitivas, nos permiten acceder a las teorías que los sujetos tienen sobre un determinado dominio y a las acciones que los lectores reconocen en el nivel metacognitivo (Karmiloff-Smith, 1997).

Las características de la interacción entre ambas dimensiones se plantea con la finalidad de proponer una tipologización del estudiante-lector que ingresa a la universidad, esta caracterización permitirá reconocer los elementos que caracterizan las teorías implícitas de los estudiantes. Así, esta caracterización permitirá reconocer los elementos propios de las teorías implícitas de los estudiantes, tanto como la relación que se establece con su conocimiento metacognitivo. Adicionalmente, interesa observar las particularidades referidas a los ámbitos del conocimiento de los estudiantes, los que han sido distribuidos en cuatro grupos de formación que representan las comunidades discursivas en que se insertan (Parodi, 2005).

En este sentido, las nociones de teoría implícita y de conocimiento metacognitivo permiten abordar, desde sus supuestos teórico-metodológicos, un conjunto de conocimientos del lector que resultan necesarios de explicitar y sistematizar. De este modo, es posible enfrentar las deficiencias en el ámbito de la comprensión textual con una caracterización del lector integradora (González, 2008; Rodríguez, 2001), permitiéndonos analizar la competencia lectora como una red compleja de múltiples procesos, en donde la experiencia y los modelos culturales cumplen un importante rol.

\section{TEORÍAS IMPLÍCITAS: UN ENFOQUE DE LA COMPRENSIÓN TEXTUAL}

Como una forma de aproximarnos a la comprensión textual y abordar los diversos factores relacionados con las competencias lectoras, se plantea el estudio de las teorías implícitas que sostienen los lectores acerca de la comprensión de textos. Las teorías o constructos epistémicos de representación de la realidad orientarían las acciones de los sujetos en diversos dominios de la vida cotidiana (Castro, 2006; Pozo, 2001; Rodrigo y Correa, 2001; Pozo y Carretero, 1987). En esta línea, se plantea que las personas presentarían 'sistemas de experiencias de dominios', a partir de los cuales sintetizarían una determinada teoría para diferentes propósitos en determinados momentos (Marrero, 2009, 1988; Pozo, Scheuer, Mateos y Pérez Echeverría, 2006; Pozo, Pérez, Sanz y Limón, 1992).

Producto de lo anterior, las teorías asumidas por los sujetos no responderían a prototipos puros, sino que constituirían síntesis que combinarían información procedente de diversas fuentes (entorno sociocultural, experiencia, formación escolar). Al mismo tiempo, y dado el carácter situado de estas representaciones, su naturaleza es esencialmente dinámica y, en términos del procesamiento cognitivo, se activarían como redes de trazos en respuesta a una demanda cognitiva (Martínez, 2009; Pozo y Rodrigo, 2001).

Las teorías implícitas nos proporcionarían respuestas a preguntas de diversa naturaleza, resituando el conocimiento cotidiano desde un nuevo marco comprensivo (Jiménez, 2002; Rodrigo y Correa, 2001; Rodríguez, 2001). Asimismo, se destaca su carácter implícito, personal y encarnado, constituyendo un saber hacer más que un saber decir, por lo mismo varios autores destacan su carácter procedimental (Pozo et al., 2006; Pozo, 2003; Pozo y Rodrigo, 2001). No obstante lo anterior, y aun cuando desde el punto de vista cognitivo constituyen sistemas diferentes (carácter implícito/procedimental v/s explícito/ 
declarativo), no funcionarían de modo independiente, por lo que destacan la necesidad de coordinar ambos sistemas de representación o conocimiento reduciendo la distancia entre la verbalización y la acción.

El análisis de las teorías implícitas plantea que éstas serían representaciones individuales construidas sobre la base de experiencias adquiridas en entornos sociales, que a su vez contienen información sobre la estructura causal, temporal e intencional de la situación (Makuc, 2013; Gómez y Guerra, 2012; Estrada, Oyarzún e Yzerbit, 2007; Rodrigo, 1997, 1994a, 1994b, 1993, 1985). De este modo, las teorías implícitas permitirán responder activamente a las diversas y variables demandas cognitivas de la situación, por cuanto la información integrada en un modelo mental de la situación facilita, por su naturaleza dinámica (en contraposición a los esquemas), la posibilidad de incrementarse y modificarse mediante una permanente negociación de significados en contextos diversos, esto es, en el marco de un proceso de intercambio social, de las exigencias profesionales, del grado de experticia y/o de redescripción representacional (Marrero, 2009; Pozo, 2001; Rodrigo, Rodríguez y Marrero, 1993).

La comprensión de textos ha constituido un tema de investigación que ha sido abordado desde numerosos enfoques (Parodi, 2009, 2007, 2005; Ruiz, 2009; González, 2008; Velásquez, Cornejo y Roco, 2008; Carlino, 2003; Peredo, 2003; Bahloul, 2002). Entre ellos, el estudio de las teorías implícitas del lector ha permitido sistematizar las teorías de los estudiantes en los distintos niveles educativos. En ellos llama la atención la importante influencia de teorías reproductivas, lineales o basadas en el procesamiento perceptual; en términos globales, se observa que los estudiantes no han activado teorías de la comprensión que permitan un desarrollo de estrategias cognitivas o metacognitivas para lograr los objetivos del lector, al contrario, en los estudiantes se observan concepciones del lector, del texto y de la comprensión que activarían acciones y procedimientos propios de un lector inexperto (Makuc, 2012, 2011; Hernández, 2008; Vélez, 2006; Peronard et al., 2002, 2000; Rivera, 2000; Schraw, 2000; Schraw \& Bruning, 1996).

En términos generales, las teorías implícitas sobre la comprensión textual representan modelos de transmisión que promueven concepciones reproductivas y lineales de la lectura, en oposición a los modelos transaccionales, basados en una concepción interactiva y constructivista de la comprensión (More y Narciso, 2011; Schraw, 2000; Schraw \& Bruning, 1996). Por otra parte, los estudios muestran, además, que las teorías sobre la comprensión experimentan un proceso de cambio, pues los estudiantes evolucionan desde una concepción lineal hacia una concepción interactiva, cambio que suele relacionarse con mayores niveles de experticia lectora y una progresiva complejización de las estrategias lectocomprensivas (Peronard, 2005, 2002; Makuc, 2010, 2009).

En el contexto de nuestro estudio, los resultados obtenidos a partir del desarrollo del constructo de teorías implícitas aplicado a la comprensión de textos han permitido identificar tres teorías sobre la comprensión: Teoría Lineal, Teoría Interactiva y Teoría Literaria. Dichas teorías se analizan en relación con tres nociones fundamentales para la comprensión de textos: noción de comprensión, de lector y de texto. Esta clasificación se ha realizado integrando información desde estudios historiográficos que sistematizan los diversos enfoques en el desarrollo de este concepto. Posteriormente, estudios cualitativos y cuantitativos han permitido proponer en forma sintética los supuestos de cada teoría en torno a la noción de comprensión, de lector y de texto. La presencia de estas teorías en el ámbito educativo, tanto en docentes secundarios como en estudiantes universitarios, se describe y analiza en diversos estudios (Makuc, 2013, 2012, 2011, 2010, 2008). 


\subsection{ESTRATEGIAS METACOGNITIVAS Y COMPRENSIÓN DE TEXTOS}

La relación entre teorías implícitas sobre compresión y estrategias metacognitivas nos permite abordar dos aspectos fundamentales que forman parte del conocimiento que el lector activa cuando se enfrenta a tareas cognitivas en el procesamiento de textos. Como es sabido, los estudios sobre metacognición distinguen entre el conocimiento y el control de la propia actividad cognitiva, uno de los supuestos básicos que se ha adoptado desde esta perspectiva es que ambas facetas son importantes para el aprendizaje y están en estrecha relación, de modo que el aprendiz competente emplea sus conocimientos metacognitivos para autorregular eficazmente su aprendizaje y, a su vez, la regulación que ejerce sobre el propio aprendizaje puede llevarle a adquirir nuevos conocimientos relacionados con la tarea y con las estrategias para afrontarla.

El conocimiento metacognitivo, considerado de naturaleza declarativa al igual que nuestro conocimiento en cualquier otro dominio, es un conocimiento explicitable que se desarrolla con la edad y la experiencia. Por su parte, el control metacognitivo tendría, en cambio, un carácter procedimental, pues se relacionaría con el control activo de los recursos disponibles expresándose en un funcionamiento eficaz del sujeto en una determinada tarea. En relación al grado de conciencia que se puede observar entre conocimiento y control, planteamientos recientes han abandonado la idea de la separación entre metacognición consciente y metacognición no consciente, para sustituirla por la idea de la progresiva explicitación del conocimiento sobre la propia actividad cognitiva. Acorde con estos planteamientos, el desarrollo metacognitivo avanzaría desde un conocimiento más implícito hasta un conocimiento más explícito (Pozo et al., 2006).

En los estudios evolutivos de la metacognición de la lectura (metacomprensión), Peronard (2000) revisa una serie de instrumentos y estrategias para acceder a este tipo de conocimiento. La autora observa que todas estas mediciones parten del supuesto de que la metacognición es consciente y asequible mediante la introspección, sin embargo, plantean interrogantes acerca del carácter consciente del monitoreo y de los otros mecanismos de autorregulación, considerando que la sensación de fallo en la comprensión puede no ser lo suficientemente consciente como para ser verbalizable, requisito que se suele considerar como evidencia de que cierto estado o proceso cognitivo ha llegado a la conciencia. En oposición a este planteamiento, se reafirma la distinción entre cognición (inconsciente y automática) y metacognición (consciente y deliberada), observación que alude al control metacognitivo, es decir, a la aplicación de estrategias remediales una vez detectado un fallo en la comprensión.

Los estudios sobre metacognición han constituido los principales fundamentos de la aproximación al conocimiento metacomprensivo. En consecuencia, a partir de las propuestas de diversos autores (Paris, Lipson \& Wixon, 1983), se plantea abordar el conocimiento del lector aplicando la noción de metacognición. Asumiendo la complejidad de los procesos lectores, el estudio del conocimiento metacomprensivo realizado por diversos investigadores ha excluido el estudio del conocimiento procedimental y se ha centrado en el estudio del conocimiento declarativo (Peronard, 2000), ello debido a la imposibilidad de verificar una serie de procesos cognitivos automatizados y relacionados con la construcción de significados durante la lectura, entre ellos, la activación de conocimientos previos, las inferencias, la generación de la base textual, etc.

Estos estudios incorporan el saber metalingüístico, en otras palabras, el saber textual del lector, de este modo han propuesto distinguir entre estrategias de comprensión, 
estrategias de evaluación, estrategias remediales, teoría del texto y de la lectura. Como podemos observar, este último tipo de conocimiento se relacionaría estrechamente con las estrategias cognitivas lectocomprensivas, por cuanto el conocimiento condicional, propio del conocimiento metacognitivo, le permitiría al lector saber cuándo y para qué activar determinada estrategia, así como qué teoría del texto y de la lectura resultan más eficaces.

\subsection{TEORÍAS IMPLÍCITAS Y ESTRATEGIAS METACOGNITIVAS: ELEMENTOS DE LA REDESCRIPCIÓN REPRESENTACIONAL}

La explicitación del conocimiento de los lectores respecto de la comprensión de textos se enmarca en la propuesta de la teoría de la redescripción representacional. Esta teoría sostiene que en el conocimiento existen niveles que fluctúan entre lo implícito (nivel procedimental) y lo explícito (nivel declarativo). Al respecto, ha habido varios intentos por definir la polaridad implícito/explícito como un continuo más que como una dicotomía (Dienes \& Perner, 1999; Karmiloff-Smith, 1997). Frente a esta dicotomía proponen la necesidad de establecer distintos niveles en el continuo explícito/implícito, respondiendo a criterios ya sea representacionales, funcionales o conceptuales. La idea que subyace a estas propuestas es que la explicitación es un proceso gradual, y que en todo conocimiento hay componentes implícitos y explícitos. Desde un punto de vista teórico se ha distinguido entre verbalización y reconocimiento, lo que representaría niveles de explicitación que permitirían dar cuenta de nuestros conocimientos acerca de un dominio determinado. Karmiloff-Smith (1984), a través de la teoría de la redescripción representacional, intenta conciliar el enfoque innatista modularista (Fodor, 1995) con el enfoque piagetano sobre la construcción generalizada del conocimiento.

En esta propuesta existiría predisposición innata específica, correspondiente a ciertos microdominios que no pueden definirse como módulos encapsulados, sino más bien como un "sesgo o boceto en esqueleto" (Karmiloff-Smith, 1997) que, progresivamente, y debido a factores externos e internos, experimentarían un proceso de modularización haciéndose menos accesibles, más automáticos e independientes de otros sistemas. La idea central de la teoría de la redescripción representacional es que, junto a este proceso de encapsulamiento, se produce un proceso inverso de mayor apertura y accesibilidad. Gracias a los procesos mediante los cuales el sujeto va cambiando el formato de representación de la información que ha almacenado, ésta pasa de niveles implícitos (conocimiento procedimental) a niveles más explícitos y más conscientes.

En el contexto de nuestra investigación se ha incorporado la propuesta de redescripción representacional, considerando que los elementos que constituyen las teorías implícitas de los estudiantes sobre la comprensión, así como las estrategias metacognitivas que ellos reconocen, se situarían dentro de un continuo representacional entre el nivel implícito y el nivel explícito de los conocimientos del lector respecto de la comprensión textual. Este proceso se aborda considerando tres componentes centrales de la comprensión textual: la noción de comprensión, de lector y de texto.

Ambos instrumentos las explicitan, tanto desde las teorías implícitas respecto de estos tópicos, como desde las estrategias del lector. De hecho, ambos instrumentos se sustentan en tres teorías de base, las que a su vez presentan coincidencias respecto de sus supuestos y de los enunciados que describen dichos componentes. No obstante sus similitudes, 
ambos instrumentos abordan la comprensión de textos desde modelos teóricos diferentes. Por una parte, interesa determinar teorías que subyacen a las afirmaciones de los lectores $\mathrm{y}$, por otra parte, las afirmaciones nos dan cuenta de las estrategias metacognitivas del lector, que aunque se sostienen en teorías, lo relevante de este constructo es determinar la experticia lectora a partir de las estrategias metacognitivas.

Este proceso de redescripción representacional es fundamental para acceder a los diversos formatos en que el conocimiento del lector se presenta. En el ámbito de la comprensión textual nos parece fundamental abordar el estudio del conocimiento del lector, explicitando dichos formatos en sus diversos niveles y con variados instrumentos. Por esta razón, en el nivel E2 (de reconocimiento) hemos explicitado mediante dos instrumentos diferentes las teorías de la comprensión y las estrategias metacognitivas, pues, por un lado, accedemos a las teorías que lo identifican como lector y, por otro lado, a aquellas estrategias que el lector reconoce como las más utilizadas en el marco de la lectura comprensiva.

\section{METODOLOGÍA}

Para llevar a cabo este estudio nos hemos situado en el contex to de estudiantes universitarios de primer año, centrándonos en dos propósitos simultáneamente. En primer lugar, interesa identificar las teorías implícitas sobre comprensión de textos que predominan en estudiantes universitarios al ingreso a la universidad. En segundo lugar, se indaga en las estrategias metacognitivas del estudiante que adhiere a las diversas teorías implícitas en uso en el ámbito de su formación universitaria. De este modo, tanto las teorías implícitas como las estrategias metacognitivas de la comprensión textual se analizan en el contexto de los grupos de formación propios del contexto académico universitario. Dados estos objetivos, se realiza un estudio exploratorio descriptivo orientado a caracterizar a los estudiantes a partir de las teorías implícitas, determinando la presencia de estas teorías en los estudiantes de primer año.

\subsection{MUESTRA}

El universo o población de estudiantes considerados en la presente investigación corresponde a aquellos matriculados de primer año vía PSU (año 2012), esto es, 386 alumnos. Estos estudiantes corresponden mayoritariamente a alumnos egresados de colegios municipalizados y particulares subvencionados (75\%) de la Región de Magallanes y Antártica Chilena.

La muestra final del estudio está constituida por 264 estudiantes de primer curso universitario que participaron voluntariamente en la investigación. El tamaño muestral es representativo de la población al nivel de confianza del 95\%. Proceden de las siguientes facultades: Facultad de Humanidades, Ciencias Sociales y de la Salud, Facultad de Ciencias Económicas, Administrativas y Jurídicas, Facultad de Ciencias y Facultad de Ingeniería. Dado que uno de los objetivos del estudio es determinar diferencias de acuerdo al ámbito de formación del estudiante, los estudiantes se han reagrupado en relación con su disciplina. La tabla siguiente presenta a los estudiantes participantes organizados en los cuatro grupos de formación empleados en los análisis. 
Estudios Pedagógicos XLI, Nº 1: 143-166, 2015

LAS TEORÍAS IMPLÍCITAS SOBRE LA COMPRENSIÓN TEXTUAL Y LAS ESTRATEGIAS METACOGNITIVAS DE ESTUDIANTES UNIVERSITARIOS DE PRIMER AÑO

Tabla $N^{\circ} 1$. Distribución de estudiantes según grupos de formación

\begin{tabular}{|c|c|c|}
\hline & Grupos de formación & $\mathbf{N}^{\circ}$ \\
\hline Educación & $\begin{array}{l}\text { Ciencias Sociales y Educación: Psicología, Trabajo Social, Pedagogía } \\
\text { Inglés, Básica y Castellano. }\end{array}$ & 88 \\
\hline Salud & Ciencias de la Salud: Enfermería, Terapia Ocupacional y Kinesiología. & 91 \\
\hline Economía & $\begin{array}{l}\text { Ciencias Económicas, Administrativas y Jurídicas: Derecho, Ingeniería } \\
\text { Comercial. }\end{array}$ & 56 \\
\hline \multirow[t]{2}{*}{ Ingeniería } & Ciencias de la Ingeniería: Ingeniería Plan Común. & 29 \\
\hline & TOTAL & 264 \\
\hline
\end{tabular}

Fuente: elaboración propia.

\subsection{INSTRUMENTOY PROCEDIMIENTOS}

Como una forma de lograr los objetivos de investigación, se elaboró un cuestionario para medir las teorías implícitas de comprensión lectora en los estudiantes universitarios. El cuestionario está constituido por 27 ítems, 9 por cada teoría (lineal, interactiva y literaria). Ello dio origen a un instrumento que, siendo unitario, puede ser desagregado en tres sub-escalas de teorías implícitas. Cabe señalar que los ítems fueron elaborados incluyendo contenidos teóricos de los tres conceptos centrales en la comprensión de textos (comprensión, lector y texto).

En primer lugar, la sub-escala caracterizada como Teoría Lineal está compuesta por 9 enunciados que implican una concepción de la comprensión vinculada a la decodificación de signos gráficos, extracción de información relevante, reconocimiento léxico, interpretación de sentidos y reproducción literal del texto. Por ejemplo: "Considero que la comprensión sólo es posible si se interpreta la totalidad de las ideas que el autor plantea".

En segundo lugar, la sub-escala caracterizada como Teoría Interactiva está compuesta por 9 enunciados que implican una concepción de la comprensión que supera el procesamiento lineal, en el cual el lector debe interpretar los significados integrando sus conocimientos previos. Por ejemplo: "Creo que los lectores hábiles confían menos en el factor visual y recurren principalmente a los conocimientos que poseen acerca de lo que leen".

En tercer lugar, la sub-escala caracterizada como Teoría Literaria está compuesta por 9 enunciados que implican concebir la comprensión basada en la valoración estética de un texto, en la identificación de personajes, temas y problemas humanos. Por ejemplo: "Comprender es imaginar, disfrutar, valorar estéticamente un texto, para que haya comprensión debe existir empatía entre el lector y los personajes".

Para acceder a los sujetos de estudio se solicitó a los Jefes de las Carreras de Pregrado su autorización respectiva para aplicar ambos cuestionarios al inicio del año académico (marzo 2012), específicamente, en algunos espacios formativos que permitían realizar esta actividad de investigación. En este contexto de indagación, se solicitó a los estudiantes que 
informaran sobre su grado de acuerdo con la información contenida en cada ítem en una escala tipo Likert de 5 puntos (1-nada, 2-poco, 3-algo, 4-bastante, 5-mucho).

Desde el punto de vista estadístico es relevante informar que el cuestionario de teorías implícitas ha alcanzado significativos índices de fiabilidad en todas las sub-escalas: $\alpha=.61$ para la Teoría Lineal, $\alpha=.71$ para la Teoría Interactiva, y $\alpha=.73$ para la Teoría Literaria.

\section{RESULTADOS DE INVESTIGACIÓN}

En esta sección se presentan los resultados generales obtenidos en la aplicación de los instrumentos diseñados para aproximarse tanto a las teorías implícitas que sostienen los estudiantes universitarios de primer año, como a las estrategias metacognitivas que aplican para la comprensión de textos escritos en sus respectivos contextos formativos. En relación con los valores estadísticos más relevantes obtenidos, se verifica que las tres teorías implícitas presentan correlaciones significativas entre ellas. Por ejemplo, entre la Teoría Lineal y la Teoría Interactiva se alcanza una correlación de .636 $(p<.000)$, con la Teoría Literaria la correlación es de .474 ( $p<.000)$, respecto de la asociación entre la Teoría Interactiva y la Teoría Literaria la correlación es de .611 $(p<.000)$.

Desde el punto de vista de los estadígrafos de tendencia central, en la siguiente tabla se presentan los resultados obtenidos por los estudiantes al contestar el Cuestionario de Teorías Implícitas para los diversos grupos formativos en estudio:

Tabla $N^{\circ}$ 2. Puntuaciones medias según el grupo de estudio

\begin{tabular}{|l|c|c|c|}
\hline & Teoría Lineal & Teoría Interactiva & Teoría Literaria \\
\hline Educación & 2.94 & 2.87 & 2.85 \\
\hline Salud & 3.08 & 3.04 & 2.96 \\
\hline Economía & 2.91 & 2.76 & 2.61 \\
\hline Ingeniería & 3.04 & 2.85 & 2.74 \\
\hline
\end{tabular}

Fuente: elaboración propia.

Como se puede observar en las puntuaciones medias obtenidas por los estudiantes en cada una de las tres sub-escalas del cuestionario, y de acuerdo a sus respectivas disciplinas de formación, predomina la aceptación de la Teoría Lineal, alcanzando los valores medios más altos en todas las disciplinas y destacándose el área de Salud en el límite superior de la distribución. Una reafirmación de esta distribución se observa en los diagramas de caja de la siguiente figura. Se puede apreciar gráficamente cómo los cuatro grupos de estudio se distribuyen homogéneamente en relación a la sub-escala de la Teoría Lineal. 
Figura $N^{\circ} 1$. Diagramas de caja para sub-escala Teoría Lineal

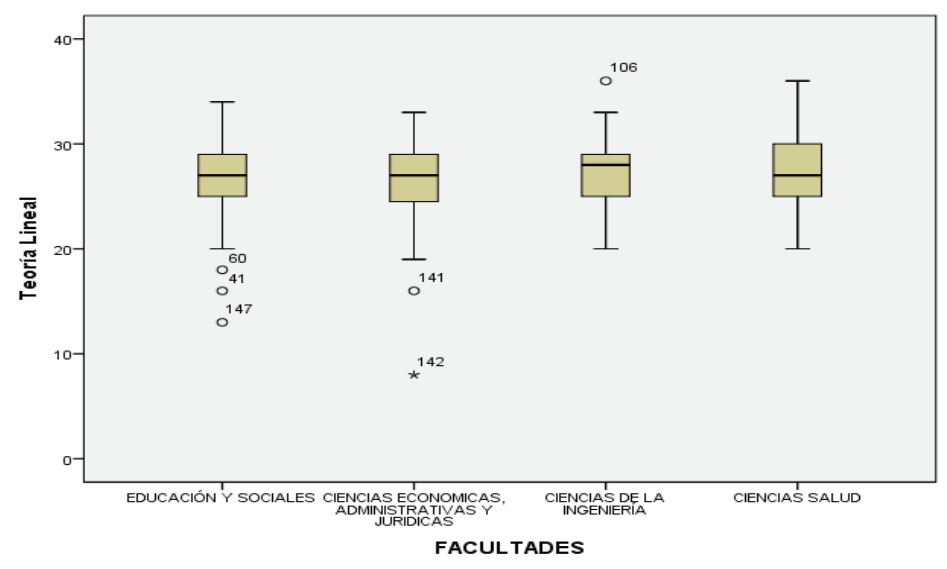

Fuente: elaboración propia.

En la Figura $\mathrm{N}^{\circ} 1$ se aprecia que la distribución de los puntajes medios en los grupos de formación perteneciente a Ciencias de la Salud y Educación es bastante similar entre sí, aun cuando en el grupo de Educación se produce mayor número de casos extremos referidos a puntaciones bajas. Adicionalmente, en el grupo de Ingeniería se observa que el $50 \%$ de los puntajes promedios se polariza hacia el límite superior de la sub-escala lineal. A pesar de ello, en los cuatros grupos se aprecian similitudes.

Desde una perspectiva inversa de análisis, las diferencias inter-grupos más significativas se encuentran en el desempeño de la sub-escala de la Teoría Literaria, lo cual se puede apreciar en la siguiente figura:

Figura $N^{\circ}$ 2. Diagramas de caja para sub-escala Teoría Literaria

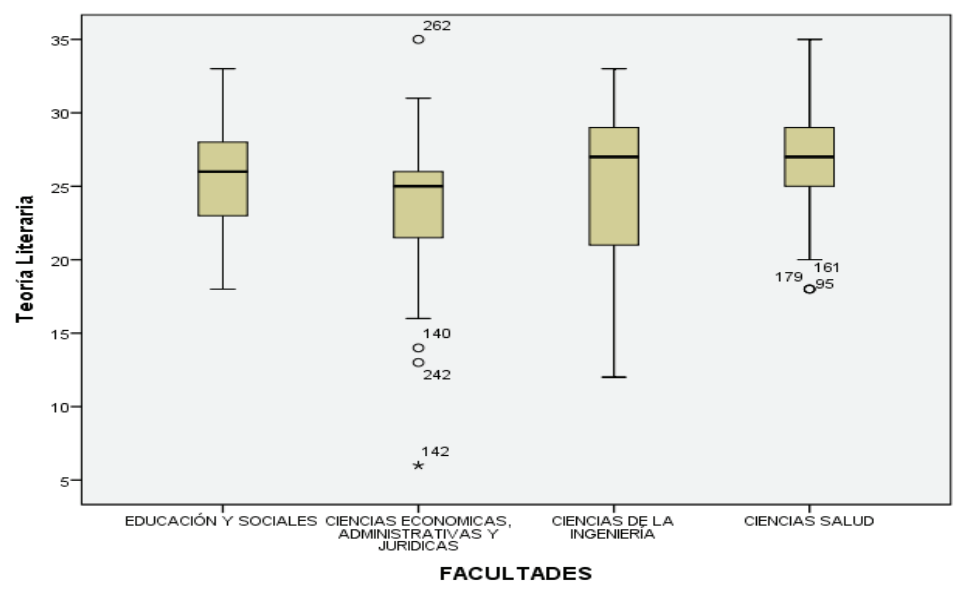

Fuente: elaboración propia 
En la Figura $\mathrm{N}^{\circ} 2$ se observa una mayor diferencia entre grupos, la distribución es disímil entre sí, sobre todo aquella que corresponde a los estudiantes de Ciencias de la Ingeniería. Al interior de este grupo de formación existen puntajes muy heterogéneos. Por su parte, el promedio menor se encuentra la Facultad de Ciencias Económicas y, por el contrario, Ciencias de la Salud presenta el mayor puntaje promedio.

Por otra parte, los análisis multivariantes indican la influencia del grupo de estudio, $F=2.77, p<.005$ y de la intersección, $F=3816.47, p<.000$. El modelo corregido obtiene un $R^{2}=.065$. En la sub-escala de la Teoría Lineal las diferencias no llegan a alcanzar significación estadística.

Tabla $N^{\circ}$ 3. Contrastes de medias según los grupos de estudio

\begin{tabular}{|c|c|c|c|c|c|}
\hline & $M$ & $S x$ & $F$ & $p$ & Eta \\
\hline \multicolumn{6}{|c|}{ Teoría Lineal } \\
\hline Educación & 2.94 & 0.41 & \multirow{4}{*}{2.27} & \multirow{4}{*}{.081} & \multirow{4}{*}{.161} \\
\hline Salud & 3.08 & 0.39 & & & \\
\hline Economía & 2.91 & 0.62 & & & \\
\hline Ingeniería & 3.04 & 0.40 & & & \\
\hline \multicolumn{6}{|c|}{ Teoría Interactiva } \\
\hline Educación & 2.87 & 0.42 & \multirow{4}{*}{4.78} & \multirow{4}{*}{.003} & \multirow{4}{*}{.230} \\
\hline Salud & 3.04 & 0.39 & & & \\
\hline Economía & 2.76 & 0.62 & & & \\
\hline Ingeniería & 2.85 & 0.40 & & & \\
\hline \multicolumn{6}{|c|}{ Teoría Literaria } \\
\hline Educación & 2.86 & 0.41 & \multirow{4}{*}{5.97} & \multirow{4}{*}{.001} & \multirow{4}{*}{.255} \\
\hline Salud & 2.95 & 0.41 & & & \\
\hline Economía & 2.61 & 0.66 & & & \\
\hline Ingeniería & 2.74 & 0.60 & & & \\
\hline
\end{tabular}

Fuente: elaboración propia.

Para conocer cómo se distribuyen los estudiantes atendiendo las teorías implícitas de comprensión según los grupos de estudio, hemos segmentado la muestra en tres niveles para cada una de las teorías implícitas de comprensión: bajo, medio y alto. Para obtener dicha segmentación hemos calculado los valores en la muestra total realizando tres grupos iguales porcentualmente. 
No existen diferencias en la distribución de la Teoría Lineal, $\chi^{2}=4.93, p=.552$, el $70 \%$ de los estudiantes de todos los grupos de estudio se ubica en los niveles medio y alto, predominan en el nivel alto los estudiantes de Ingeniería, 51.7\%. Tampoco se producen diferencias significativas en la distribución de la Teoría Interactiva, $\chi^{2}=10.43, p=.108$. Sin embargo, en la Teoría Literaria sí que aparecen diferencias, $\chi^{2}=26.55, p<.000$, en la dirección de mayores porcentajes del nivel bajo en los estudiantes de Economía e Ingeniería, el $73 \%$ de los estudiantes de Educación se ubica entre los niveles medio y alto. Solamente el 19\% de los estudiantes de Salud se sitúa en el nivel bajo.

\subsection{CUESTIONARIO DE METACOMPRENSIÓN}

El Cuestionario de Metacomprensión es un instrumento que se orienta al reconocimiento de alternativas relacionadas con el dominio de la comprensión textual, en un nivel del conocimiento menos explícito que ha sido definido como E2 (Karmiloff-Smith, 1997). Para la cuantificación de los resultados se han asignado puntajes diferenciados a las diversas alternativas según el grado en que éstas reflejan conocimiento de la tarea, del texto y de las estrategias de un experto ideal.

Las alternativas que implican un conocimiento de la lectura como proceso interactivo en un contexto comunicativo obtienen 30 puntos; las alternativas que reconocen el texto como un todo coherente y semánticamente jerarquizado obtienen 20 puntos; se asignan 12 puntos a las alternativas en las que se enfatiza un conocimiento afectivo-actitudinal; se asignan 9 puntos a las alternativas que implican una teoría de la tarea cercana a la memorización y una teoría del texto muy centrada en aspectos externos; se asignan 4 puntos a las alternativas que implican un conocimiento muy rudimentario, vago e impreciso del texto y de la tarea, y, finalmente; se asigna 1 punto a la alternativa "No lo he pensado" que indica ausencia de metaconocimiento, y, por tanto, inexistencia de una teoría acerca de la lectura comprensiva. La cuantificación se ha basado en las siguientes teorías de la tarea y del texto sintetizadas en la tabla que se presenta a continuación:

Tabla $N^{\circ}$ 4. Resumen teorías de base según Cuestionario de Metacomprensión

\begin{tabular}{|c|l|c|}
\hline $\begin{array}{c}\text { PUNTUACIONES } \\
\text { \% PERICIA }\end{array}$ & TEORÍAS DE BASE \\
\hline 1 & Despreocupación total por el tema & 0 \\
\hline 4 & Teoría superficial e imprecisa & 30 \\
\hline 9 & Teoría basada en lo externo y físico & 40 \\
\hline 12 & Teoría de índole afectiva y actitudinal & 60 \\
\hline 20 & Teoría del "traspaso" del contenido al lector & 100 \\
\hline 30 & Teoría interactiva/comunicativa/pragmática & \\
\hline
\end{tabular}

Fuente: elaboración propia. 


\subsection{RESULTADOS CUESTIONARIO DE METACOMPRENSIÓN}

Una visión global de los resultados nos permite comprobar que el mayor porcentaje de respuestas $(21,3 \%)$ se concentra en el puntaje 20 , el que se relaciona con una concepción de la lectura centrada en el traspaso del contenido al lector y se corresponde con un $60 \%$ de experticia. El análisis desagregado de cada componente nos muestra que el componente planificación (25\%) obtiene mayor número de respuestas en el puntaje 30 y en el puntaje 12 con el 24,4\% de las respuestas. En segundo término, el componente evaluación (23,9\%) concentra el mayor porcentaje de respuestas en el puntaje 20, el componente comprensión también presenta un porcentaje mayor de respuestas $(22,9 \%)$ en el puntaje 20 , así como el componente tarea $(22,2 \%)$ que concentra una buena cantidad de respuestas en este puntaje. En tercer lugar, el componente texto $(21,8 \%)$ recibe el mayor porcentaje de respuestas en el puntaje 12. Finalmente, el componente remedial $(21,1 \%)$ obtiene mayor porcentaje de respuestas en el puntaje 20.

Como podemos observar en la tabla siguiente, los componentes de planificación y evaluación de la lectura concentran un mayor porcentaje de respuestas vinculadas a estrategias metacomprensivas de un lector experto, mientras que el componente texto concentra mayor porcentaje en puntaje 12 que implica una teoría sobre el texto asociada a niveles bajos de experticia.

Tabla $N^{\circ} 5$. Distribución de respuestas por componente

\begin{tabular}{|l|c|c|c|c|c|c|}
\hline Componente & $\mathbf{1}$ & $\mathbf{4}$ & $\mathbf{9}$ & $\mathbf{1 2}$ & $\mathbf{2 0}$ & $\mathbf{3 0}$ \\
\hline Comprensión & $9,7 \%$ & $10,4 \%$ & $18,6 \%$ & $17,7 \%$ & $22,9 \%$ & $20,8 \%$ \\
\hline Evaluación & $7,5 \%$ & $18,4 \%$ & $10,1 \%$ & $18,0 \%$ & $23,9 \%$ & $22,0 \%$ \\
\hline Planificación & $10,2 \%$ & $8,3 \%$ & $12,8 \%$ & $24,4 \%$ & $19,3 \%$ & $25,0 \%$ \\
\hline Remedial & $9,5 \%$ & $19,2 \%$ & $16,4 \%$ & $16,6 \%$ & $21,1 \%$ & $17,2 \%$ \\
\hline Tarea & $8,8 \%$ & $17,1 \%$ & $16,8 \%$ & $14,1 \%$ & $22,2 \%$ & $20,9 \%$ \\
\hline Texto & $12,5 \%$ & $8,8 \%$ & $19,2 \%$ & $21,8 \%$ & $18,4 \%$ & $19,2 \%$ \\
\hline TOTAL & 9,7 & 13,7 & 15,6 & 18,7 & 21,3 & 20,8 \\
\hline
\end{tabular}

Fuente: elaboración propia.

El análisis de los resultados, considerando la experticia y agrupando respuestas que obtienen entre 20 y 30 puntos, es decir, entre el $60 \%$ y el $100 \%$ de experticia, junto con la revisión de los componentes, permite observar lo siguiente: las estrategias de evaluación presentan un $45,9 \%$ de respuestas en el rango mayor de experticia, y un 54,1\% de respuestas en el rango de menor experticia. Esta distribución homogénea de respuestas se presenta de manera similar en los componentes de planificación, comprensión y tarea. Esta homogeneidad no se observa en los componentes de remedial y texto, los cuales concentran un alto porcentaje de respuestas en el rango de menor experticia. En la tabla siguiente se observa el detalle: 
Estudios Pedagógicos XLI, Nº 1: 143-166, 2015

LAS TEORÍAS IMPLÍCITAS SOBRE LA COMPRENSIÓN TEXTUAL Y LAS ESTRATEGIAS METACOGNITIVAS DE ESTUDIANTES UNIVERSITARIOS DE PRIMER AÑO

Tabla $N^{\circ}$ 6. Respuestas asociadas a grados de experticia (lector experto v/s inexperto)

\begin{tabular}{|l|c|c|}
\hline Componente & $\begin{array}{c}\text { Lector experto } \\
(\mathbf{6 0 - 1 0 0 \%} \text { experticia) }\end{array}$ & $\begin{array}{c}\text { Lector inexperto } \\
(\mathbf{0 - 4 0 \%} \text { experticia) }\end{array}$ \\
\hline Evaluación & $45,9 \%$ & $54,1 \%$ \\
\hline Planificación & $44,3 \%$ & $55,7 \%$ \\
\hline Comprensión & $43,7 \%$ & $56,3 \%$ \\
\hline Tarea & $43 \%$ & $56,9 \%$ \\
\hline Remedial & $38 \%$ & $62 \%$ \\
\hline Texto & $37,6 \%$ & $63 \%$ \\
\hline TOTAL & $42 \%$ & $58 \%$ \\
\hline
\end{tabular}

Fuente: elaboración propia.

En síntesis, en términos del conocimiento metacognitivo y de las estrategias metacognitivas, los estudiantes se identifican con estrategias propias de un lector inexperto en un $58 \%$ de sus respuestas, mientras que el $42 \%$ de sus respuestas se relaciona con las estrategias de un lector experto. En este sentido, evaluación, comprensión y tarea obtienen porcentajes similares de respuestas en el rango de alta y baja experticia, sin embargo, los componentes remedial y texto destacan por su bajo desarrollo metacognitivo, en tanto las respuestas se concentran en las estrategias de un lector inexperto.

\subsubsection{Cuestionario de Metacomprensión según componentes teóricos y desempeño de los grupos de formación}

En esta sección se presentan los resultados de la aplicación del Cuestionario de Metacomprensión desagregados de acuerdo a sus distintos componentes estructurales que lo integran: tarea, planificación, evaluación, estrategia, remedial y texto. El análisis detallado de los resultados obtenidos en cada uno de dichos componentes se realiza, a su vez, explorando su relación con las nociones teóricas de base (comprensión, lector y texto), que estructuran o conforman las teorías implícitas sobre la comprensión de textos por parte de los estudiantes universitarios de primer año respecto de cada componente.

La noción de comprensión se describe a través del primer componente estratégico definido como tarea. En segundo término, se presentan los elementos correspondientes a la noción de lector, y se explicita su relación con los siguientes componentes de la metacomprensión que configuran a un lector como estratégico: planificación, estrategia, evaluación y remedial. Por último, la noción de texto emerge como una categoría con una estructura propia. En síntesis, este cuestionario distingue en la categoría de lector cuatro componentes estratégicos: planificación, estrategia, evaluación y remedial. En términos cuantitativos, se expresa que el mayor número de preguntas (16 preguntas) del Cuestionario de Metacomprensión están referidas a la noción de lector. 


\subsubsection{Resultados del componente tarea}

El componente tarea corresponde a un prerrequisito del conocimiento condicional, el cuestionario contiene siete preguntas orientadas a identificar el grado de conocimiento de la tarea, es decir, de la comprensión de textos escritos. En la siguiente tabla se presentan los datos referidos al tema Conocimiento de la Tarea del Cuestionario de Metacomprensión. El componente tarea analizado por los estudiantes organizados en grupos de formación permite observar que los puntajes más altos se concentran en los estudiantes de Ciencias Sociales y Educación y Ciencias de la Ingeniería, esto los ubica mayoritariamente en el puntaje 20 que corresponde al nivel de experticia de $60 \%$.

Tabla $N^{\circ}$ 7. Componente tarea por grupo de formación

\begin{tabular}{|l|c|c|c|c|c|c|}
\hline & \multicolumn{7}{|c|}{ Componente Tarea } \\
\hline Grupos de formación & $\mathbf{1}$ & $\mathbf{4}$ & $\mathbf{9}$ & $\mathbf{1 2}$ & $\mathbf{2 0}$ & $\mathbf{3 0}$ \\
\hline Experticia & $\mathbf{0 \%}$ & $\mathbf{1 0 \%}$ & $\mathbf{2 0 \%}$ & $\mathbf{4 0 \%}$ & $\mathbf{6 0 \%}$ & $\mathbf{1 0 0 \%}$ \\
\hline $\begin{array}{l}\text { Ciencias Sociales y } \\
\text { Educación }\end{array}$ & $9,80 \%$ & $16,00 \%$ & $15,70 \%$ & $13,50 \%$ & $\underline{23,70 \%}$ & $21,20 \%$ \\
\hline Ciencias de la Salud & $8,50 \%$ & $18,10 \%$ & $17,00 \%$ & $13,90 \%$ & $21,20 \%$ & $21,20 \%$ \\
\hline $\begin{array}{l}\text { Ciencias Económicas, } \\
\begin{array}{l}\text { Administrativas y } \\
\text { Jurídicas }\end{array}\end{array}$ & $8,50 \%$ & $17,40 \%$ & $17,40 \%$ & $14,90 \%$ & $20,90 \%$ & $20,90 \%$ \\
\hline $\begin{array}{l}\text { Ciencias de la } \\
\text { Ingeniería }\end{array}$ & $7,50 \%$ & $16,70 \%$ & $18,30 \%$ & $15,00 \%$ & $\underline{23,30 \%}$ & $19,20 \%$ \\
\hline
\end{tabular}

Fuente: elaboración propia.

Como se observa en la Tabla $\mathrm{N}^{\circ} 7$, es preciso señalar que aun cuando el porcentaje mayor de respuestas de estudiantes de los dos grupos destacados en este componente (Ciencias Sociales e Ingeniería) alcanza un $60 \%$ de experticia, esto es el $47 \%$, existe aproximadamente un 55\% de los estudiantes que en estos grupos se encuentra en niveles de experticia inferiores al $40 \%$ de logro. La importancia de este componente radica en que el conocimiento de la tarea constituye un conocimiento clave para la activación de estrategias.

\subsubsection{Resultados del componente lector: Comprensión, planificación, evaluación y remedial}

El componente lector integra las estrategias de comprensión, planificación, evaluación y remedial. En la siguiente tabla se presentan los puntajes en que se ubican mayoritariamente los estudiantes, los cuales nos permiten identificar aquellos conocimientos que forman parte de la "teoría del lector" que tienen los alumnos en cada grupo de formación. 
Estudios Pedagógicos XLI, N 1: 143-166, 2015

LAS TEORÍAS IMPLÍCITAS SOBRE LA COMPRENSIÓN TEXTUAL Y LAS ESTRATEGIAS METACOGNITIVAS DE ESTUDIANTES UNIVERSITARIOS DE PRIMER AÑO

Tabla $N^{\circ}$ 8. Componente estrategias de comprensión por grupo de formación

\begin{tabular}{|l|c|c|c|c|c|c|}
\hline & \multicolumn{5}{|c|}{ Componente Estrategias de Comprensión } \\
\hline Grupos de formación & $\mathbf{1}$ & $\mathbf{4}$ & $\mathbf{9}$ & $\mathbf{1 2}$ & $\mathbf{2 0}$ & $\mathbf{3 0}$ \\
\hline Experticia & $\mathbf{0 \%}$ & $\mathbf{1 0 \%}$ & $\mathbf{2 0 \%}$ & $\mathbf{4 0 \%}$ & $\mathbf{6 0 \%}$ & $\mathbf{1 0 0 \%}$ \\
\hline Ciencias Sociales y Educación & $7,40 \%$ & $8,70 \%$ & $17,30 \%$ & $19,80 \%$ & $\underline{24,50 \%}$ & $22,30 \%$ \\
\hline Ciencias de la Salud & $9,80 \%$ & $11,80 \%$ & $18,50 \%$ & $16,60 \%$ & $22,20 \%$ & $21,10 \%$ \\
\hline $\begin{array}{l}\text { Ciencias Económicas, } \\
\text { Administrativas y Jurídicas }\end{array}$ & $9,70 \%$ & $9,70 \%$ & $\underline{22,60 \%}$ & $16,10 \%$ & $21,50 \%$ & $20,40 \%$ \\
\hline Ciencias de la Ingeniería & $15,80 \%$ & $12,30 \%$ & $15,80 \%$ & $17,50 \%$ & $\underline{22,80 \%}$ & $15,80 \%$ \\
\hline
\end{tabular}

Fuente: elaboración propia.

En las estrategias de comprensión los estudiantes de tres grupos, a saber, Ciencias Sociales y Educación, Ciencias de la Salud y Ciencias de la Ingeniería concentran sus respuestas entre los puntajes 20 y 30, sólo los estudiantes de Ciencias Económicas, Administrativas y Jurídicas presentan un porcentaje similar de respuestas en puntaje 9, que a su vez describe mínimos niveles de experticia. Este puntaje describe estrategias de comprensión vinculadas a alternativas centradas en factores externos y/o humanos (profesor/ prueba), o textuales de superficie como la ortografía y la tipografía.

Tabla $N^{\circ}$ 9. Componente planificación por grupo de formación

\begin{tabular}{|l|c|c|c|c|c|c|}
\hline & \multicolumn{6}{|c|}{ Componente Planificación } \\
\hline Grupos de formación & $\mathbf{1}$ & $\mathbf{4}$ & $\mathbf{9}$ & $\mathbf{1 2}$ & $\mathbf{2 0}$ & $\mathbf{3 0}$ \\
\hline Experticia & $\mathbf{0} \%$ & $\mathbf{1 0 \%}$ & $\mathbf{2 0 \%}$ & $\mathbf{4 0 \%}$ & $\mathbf{6 0 \%}$ & $\mathbf{1 0 0 \%}$ \\
\hline Ciencias Sociales y Educación & $9,40 \%$ & $8,30 \%$ & $13,60 \%$ & $23,80 \%$ & $18,50 \%$ & $\underline{26,40 \%}$ \\
\hline Ciencias de la Salud & $10,20 \%$ & $10,20 \%$ & $12,20 \%$ & $24,10 \%$ & $19,00 \%$ & $\underline{\mathbf{2 4 , 4 0 \%}}$ \\
\hline $\begin{array}{l}\text { Ciencias Económicas, } \\
\text { Administrativas y Jurídicas }\end{array}$ & $11,40 \%$ & $4,80 \%$ & $14,50 \%$ & $25,90 \%$ & $20,50 \%$ & $22,90 \%$ \\
\hline Ciencias de la Ingeniería & $11,10 \%$ & $8,10 \%$ & $10,10 \%$ & $24,20 \%$ & $20,20 \%$ & $\underline{26,30 \%}$ \\
\hline
\end{tabular}

Fuente: elaboración propia. 
En el componente planificación los cuatro grupos concentran sus respuestas en el puntaje 30, que describe el nivel superior de experticia (100\%). Desde esta perspectiva, el lector planifica la lectura basándose en aspectos vinculados con la coherencia y la tipología textual, considera la intencionalidad del autor y el rol activo del lector en la construcción de los significados.

Tabla $N^{\circ}$ 10. Componente evaluación por grupo de formación

\begin{tabular}{|l|c|c|c|c|c|c|}
\hline & \multicolumn{7}{|c|}{ Componente Evaluación } \\
\hline Grupos de formación & $\mathbf{1}$ & $\mathbf{4}$ & $\mathbf{9}$ & $\mathbf{1 2}$ & $\mathbf{2 0}$ & $\mathbf{3 0}$ \\
\hline Experticia & $\mathbf{0 \%}$ & $\mathbf{1 0 \%}$ & $\mathbf{2 0 \%}$ & $\mathbf{4 0 \%}$ & $\mathbf{6 0 \%}$ & $\mathbf{1 0 0 \%}$ \\
\hline $\begin{array}{l}\text { Ciencias Sociales y } \\
\text { Educación }\end{array}$ & $7,70 \%$ & $18,60 \%$ & $10,90 \%$ & $17,30 \%$ & $23,70 \%$ & $21,80 \%$ \\
\hline Ciencias de la Salud & $8,00 \%$ & $18,70 \%$ & $10,10 \%$ & $18,70 \%$ & $23,30 \%$ & $21,20 \%$ \\
\hline $\begin{array}{l}\text { Ciencias Económicas, } \\
\text { Administrativas y Jurídicas }\end{array}$ & $7,00 \%$ & $17,40 \%$ & $9,90 \%$ & $16,90 \%$ & $\underline{24,40 \%}$ & $\underline{24,40 \%}$ \\
\hline Ciencias de la Ingeniería & $6,50 \%$ & $18,70 \%$ & $8,40 \%$ & $19,60 \%$ & $\underline{25,20 \%}$ & $21,50 \%$ \\
\hline
\end{tabular}

Fuente: elaboración propia.

En relación con las estrategias de evaluación se destaca el grupo de Ciencias Económicas, Administrativas y Jurídicas, ya que sus estudiantes concentran sus respuestas en un porcentaje superior al grupo total en los puntajes 20 y 30, los cuales describen las estrategias evaluativas de un lector con niveles superiores de experticia.

Tabla $N^{\circ} 11$. Componente remedial por grupo de formación

\begin{tabular}{|l|c|c|c|c|c|c|}
\hline & \multicolumn{7}{|c|}{ Componente Remedial } \\
\hline Grupos de formación & $\mathbf{1}$ & $\mathbf{4}$ & $\mathbf{9}$ & $\mathbf{1 2}$ & $\mathbf{2 0}$ & $\mathbf{3 0}$ \\
\hline Experticia & $\mathbf{0 \%}$ & $\mathbf{1 0 \%}$ & $\mathbf{2 0 \%}$ & $\mathbf{4 0 \%}$ & $\mathbf{6 0 \%}$ & $\mathbf{1 0 0 \%}$ \\
\hline $\begin{array}{l}\text { Ciencias Sociales y } \\
\text { Educación }\end{array}$ & $8,20 \%$ & $19,20 \%$ & $16,10 \%$ & $15,30 \%$ & $\underline{22,30 \%}$ & $18,90 \%$ \\
\hline Ciencias de la Salud & $10,50 \%$ & $18,70 \%$ & $15,80 \%$ & $17,90 \%$ & $\underline{20,50 \%}$ & $16,60 \%$ \\
\hline $\begin{array}{l}\text { Ciencias Económicas, } \\
\text { Administrativas y Jurídicas }\end{array}$ & $8,60 \%$ & $20,40 \%$ & $17,60 \%$ & $16,70 \%$ & $\underline{20,80 \%}$ & $15,80 \%$ \\
\hline Ciencias de la Ingeniería & $11,50 \%$ & $18,50 \%$ & $16,90 \%$ & $16,20 \%$ & $\underline{20,00 \%}$ & $16,90 \%$ \\
\hline
\end{tabular}

Fuente: elaboración propia. 
En el componente remedial, en cambio, ninguno de los grupos formativos se sitúa en el puntaje máximo, ya que se concentran las respuestas en el puntaje 20. No obstante ello, se observa en el grupo de Ciencias Económicas, Administrativas y Jurídicas un alto porcentaje de respuestas en el puntaje 4. En este puntaje el conocimiento de estrategias remediales se manifiesta cuando el sujeto no realiza ninguna acción cuando se enfrenta con un fallo en la comprensión.

En síntesis, es posible destacar que todos los grupos de formación presentan mayor cantidad de respuestas en puntajes altos en el componente planificación; en segundo lugar, en los grupos se observan respuestas con puntajes altos en las estrategias de evaluación y en las estrategias remediales, en estos componentes los cuatro grupos alcanzan el $60 \%$ de experticia; en tercer lugar, se ubican las estrategias de comprensión.

En relación con los grupos de formación, podemos señalar que el grupo que presenta mayor disparidad de resultados corresponde al grupo de Ciencias Económicas, Administrativas y Jurídicas, pues en este grupo un porcentaje superior responde preguntas relacionadas con niveles mínimos de experticia en estrategias de comprensión. Asimismo, se observa un alto porcentaje de respuestas con altos puntajes en el componente evaluación relacionado con mayores niveles de experticia.

\subsubsection{Resultados del componente texto}

A diferencia de los datos anteriormente presentados que correspondían a los diversos componentes de una teoría del lector (estratégico), en este apartado se indaga en características referidas exclusivamente a la teoría del texto. Esta teoría aborda aspectos vinculados con la concepción o el conocimiento del texto que poseen los sujetos en estudio, tanto si es concebido por ellos como una estructura gramatical determinada, es decir, sólo como un conjunto de letras o palabras, sin mayor estructuración, o si, por el contario, se consideran algunos elementos extragramaticales (externos del texto) que pueden tener relevancia en la comprensión.

En el componente texto las alternativas predominantes $(21,8 \%)$ se ubican en el puntaje 12 , que corresponde a respuestas que describen un $40 \%$ de experticia. Este resultado indica una diferencia entre los niveles de experticia manifestados en los anteriores componentes, en ese sentido, la teoría del texto presenta la mayor cantidad de respuestas en puntajes que se vinculan con niveles bajos de experticia. Como se observa en la tabla siguiente, los mayores porcentajes de estudiantes se han ubicado en los puntajes 9 y 12 .

Tabla $N^{\circ}$ 12. Componente texto por grupo de formación

\begin{tabular}{|l|c|c|c|c|c|c|}
\hline & \multicolumn{7}{|c|}{ Componente Texto } \\
\hline Grupos de formación & $\mathbf{1}$ & $\mathbf{4}$ & $\mathbf{9}$ & $\mathbf{1 2}$ & $\mathbf{2 0}$ & $\mathbf{3 0}$ \\
\hline Experticia & $\mathbf{0} \%$ & $\mathbf{1 0 \%}$ & $\mathbf{2 0 \%}$ & $\mathbf{4 0 \%}$ & $\mathbf{6 0 \%}$ & $\mathbf{1 0 0 \%}$ \\
\hline $\begin{array}{l}\text { Ciencias Sociales y } \\
\text { Educación }\end{array}$ & $13,90 \%$ & $8,30 \%$ & $17,40 \%$ & $\underline{21,80 \%}$ & $20,10 \%$ & $18,60 \%$ \\
\hline Ciencias de la Salud & $12,30 \%$ & $8,70 \%$ & $20,10 \%$ & $\underline{21,80 \%}$ & $17,90 \%$ & $19,30 \%$ \\
\hline
\end{tabular}




\begin{tabular}{|l|c|c|c|c|c|c|}
\hline $\begin{array}{l}\text { Ciencias Económicas, } \\
\text { Administrativas y Jurídicas }\end{array}$ & $12,50 \%$ & $8,50 \%$ & $20,50 \%$ & $\underline{21,00 \%}$ & $18,00 \%$ & $19,50 \%$ \\
\hline Ciencias de la Ingeniería & $9,50 \%$ & $11,20 \%$ & $19,80 \%$ & $\underline{22,40 \%}$ & $16,40 \%$ & $20,70 \%$ \\
\hline
\end{tabular}

Fuente: elaboración propia.

Como se puede observar en todos los grupos de formación, el componente texto presenta respuestas concentradas en mayor porcentaje en el puntaje 12, que corresponde, como ya hemos señalado, a bajos niveles de experticia (40\%). En este nivel se describe una concepción del texto centrado en aspectos superficiales, tales como extensión de párrafos y evaluación de los textos en relación a su capacidad de motivación. Todos los grupos presentan mayor cantidad de respuestas en un puntaje que se relaciona con mínimos niveles de experticia, en otras palabras, los estudiantes manifiestan una débil valoración del texto en el contexto de la lectura comprensiva.

\subsubsection{Teorías implícitas y estrategias metacognitivas}

La distribución de los estudiantes atendiendo las teorías implícitas de comprensión ha permitido segmentar la muestra en tres niveles para cada una de las teorías implícitas de comprensión: nivel bajo, nivel medio y nivel alto. De este modo, se han determinado los puntajes que obtienen en el Cuestionario de Metacomprensión los estudiantes que se agrupan en las Teorías Lineal, Interactiva y Literaria, lo que permite clasificarlos según el grado de desarrollo de estrategias metacognitivas y, a su vez, en niveles de experticia asociados a dichos puntajes.

Los resultados obtenidos por el grupo total de estudiantes nos permitieron observar que, independientemente de la teoría implícita, mayoritariamente se concentran entre los puntajes 20 y 12. Estos puntajes, como lo indica el instrumento, corresponden a $60 \%$ y $40 \%$ de experticia, respectivamente.

El análisis de cada teoría en relación al puntaje en que se agrupan los estudiantes nos muestra lo siguiente: en la Teoría Lineal, los estudiantes se ubican en un 49,8\% en el puntaje 20, mientras que los estudiantes agrupados en la Teoría Interactiva se ubican en dicho puntaje en un 52,6\%. Este puntaje se vincula con una mayor conciencia de la participación activa del lector para captar las ideas expresadas en el texto, y con un concepto de texto y de lectura más sofisticado, así también, el lector presenta una clara conciencia del esfuerzo cognitivo que se debe realizar en el proceso comprensivo, y reconoce en la noción de texto una jerarquía de ideas, más que una sumatoria de palabras. Por lo tanto, este nivel indica que el lector reconoce la necesidad de captar ideas centrales y de parafrasear como un modo de comprender la información que está en el texto.

Asimismo, observamos que los estudiantes que adhieren a la Teoría Lineal se ubican en un $50 \%$ en el puntaje 12, mientras que los agrupados en la Teoría Interactiva se ubican en un $43,4 \%$ en dicho puntaje. Este nivel incluye alternativas que indican una etapa de transición entre lo externo y lo cognitivo, metacognición emergente, experiencias metacognitivas de índole actitudinal y afectiva, también alude al uso de estrategias fijas, como "volver a leer" o a la prioridad de la memorización. Por lo anterior, el puntaje 12 corresponde a $40 \%$ de experticia lectora en estrategias metacognitivas. 
Como podemos observar, la distribución de los estudiantes de ambas teorías en los puntajes 20 y 12 es bastante homogénea, no existiendo mayores diferencias en experticia lectora asociada a estrategias metacognitivas entre el grupo lineal y el grupo interactivo. Sin embargo, esta distribución de puntajes varía considerablemente entre los estudiantes que presentan un nivel medio alto en la Teoría Literaria, pues se agrupan mayoritariamente en el puntaje 12, el cual se asocia a la teoría afectiva y actitudinal. En la siguiente tabla se observa el grado de experticia de los estudiantes:

Tabla $N^{\circ}$ 13. Puntajes asociados a las teorías implícitas de los estudiantes

\begin{tabular}{|c|c|c|c|c|c|c|c|}
\hline Teoría Implícita & $\begin{array}{c}30 \\
(100 \%)\end{array}$ & $\begin{array}{c}20(60 \%) \\
\text { "Traspaso" } \\
\text { contenidos lector }\end{array}$ & \begin{tabular}{|c|}
$12(40 \%)$ \\
Afectiva y \\
actitudinal
\end{tabular} & $\begin{array}{c}9(30 \%) \\
\text { Base } \\
\text { externa }\end{array}$ & $\begin{array}{c}4 \\
(0 \%)\end{array}$ & $\begin{array}{c}1 \\
(0 \%)\end{array}$ & Total \\
\hline Teoría Lineal & & $49,8 \%$ & $50,0 \%$ & & & & 99,8 \\
\hline Teoría Interactiva & & $52,6 \%$ & $43,4 \%$ & $3,9 \%$ & & & 99,9 \\
\hline Teoría Literaria & & $24 \%$ & $75,9 \%$ & & & & 99,9 \\
\hline
\end{tabular}

Fuente: elaboración propia.

Este resultado nos indica que la teorías implícitas lineal e interactiva no presentan una relación significativa con el desarrollo metacognitivo de los estudiantes. De este modo, tanto estudiantes lineales como interactivos se distribuyen en los puntajes 20 y 12 en porcentajes similares. A su vez, las teorías implícitas lineal e interactiva se presentan con porcentajes similares de experticia metacognitiva.

Este resultado sólo difiere en el caso de los estudiantes literarios, quienes presentan un mayor porcentaje con puntaje 12, el cual, como hemos señalado anteriormente, se vincula con la teoría de base del Cuestionario de Metacomprensión, el que plantea similares supuestos con la teoría literaria. Este puntaje corresponde al cuarto nivel e incluye alternativas que indican una etapa de transición entre lo externo y lo cognitivo, las opciones se refieren a "experiencias metacognitivas" de índole afectiva. En lo que se refiere a su teoría del texto, se ubican afirmaciones que enjuician los textos a partir de lo afectivo (es difícil lo que aburre y fácil lo que entretiene). Las alternativas referidas al conocimiento de estrategias remediales y de evaluación en este nivel hacen mención a factores afectivos, las estrategias de planificación que se ubican aquí tienen que ver con la disposición anímica y con el tipo de texto.

\section{DISCUSIÓN}

En términos generales, se verifica la presencia relativamente homogénea de las tres teorías implícitas sobre comprensión entre estudiantes universitarios de primer año: Teoría Lineal, Teoría Interactiva y Teoría Literaria. Estos resultados, no obstante, requieren ser corroborados ampliando la muestra del estudio, pues la posibilidad de agrupar a los lectores 
en torno a determinadas teorías sobre la comprensión textual, puede constituir información relevante a considerar en el conocimiento del lector.

Un aspecto a destacar lo constituye el predominio de la Teoría Lineal en todos los grupos de formación, siendo la que obtiene los valores medios más elevados en todos los grupos. Asimismo, se observa que la Teoría Interactiva no presenta diferencias significativas en la distribución de los cuatro grupos de estudiantes, a diferencia de la Teoría Literaria, cuyos resultados ubican a un elevado porcentaje de estudiantes de Educación entre los niveles medio y alto, mientas que entre los estudiantes de los grupos de Economía e Ingeniería se obtiene la media más baja.

Como hemos observado en los resultados, las Teorías Lineal y Literaria se presentan de manera clara y diferenciada entre los estudiantes. La Teoría Lineal, como hemos indicado, destaca la centralidad del texto y la búsqueda de información, en otras palabras, la necesidad de asegurar una lectura fiel a los contenidos transmitidos por el texto y el autor. Mientras que la Teoría Literaria contempla la lectura, por una parte, como un proceso orientado a textos literarios, como una actividad lúdica y de crecimiento personal, y, por otra parte, se asocia la lectura en el contexto escolar en donde el análisis literario orienta al lector de acuerdo a los parámetros de dicha teoría.

La relación entre las teorías implícitas y las estrategias metacognitivas nos muestra que los estudiantes no se diferencian en términos de experticia lectora respecto de la Teoría Lineal y la Teoría Interactiva. Esta diferencia sólo se manifiesta en los estudiantes que adhieren a la Teoría Literaria, los que mayoritariamente se agrupan en el puntaje 12. Este hecho se explicaría por cuanto el puntaje 12 se sustenta en una teoría de índole actitudinal y afectiva. El análisis de los resultados, considerando grados de experticia en la revisión de los componentes, permite observar una distribución homogénea de respuestas en el rango de mayor y menor experticia, ello se observa en las estrategias de evaluación, de planificación, de comprensión y en el componente tarea. Sin embargo, esta homogeneidad no se observa en los componentes de remedial y texto, pues en ellos se concentra un alto porcentaje de respuestas en el rango de menor experticia. Es decir, los estudiantes concentran sus respuestas en el puntaje 12 en preguntas referidas a las estrategias remediales y en las afirmaciones acerca del texto, ámbitos del conocimiento metacognitivo que alcanzan un desarrollo menor.

Estos resultados nos plantean la necesidad de ampliar el estudio con estudiantes de diferentes años en las distintas carreras para analizar la relación entre la adhesión a una determinada teoría y las estrategias metacognitivas. Igualmente, y en la línea de los estudios evolutivos del desarrollo metacognitivo del lector, se plantean nuevas preguntas de investigación a estos resultados iniciales, preguntas vinculadas con la evolución de los estudiantes en cuanto a las teorías a las que adhieren y a su relación con el desarrollo metacognitivo, especialmente como comunidad discursiva específica socializada en prácticas lectoras y en géneros discursivos académicos como parte identitaria de su formación.

\section{REFERENCIAS BIBLIOGRÁFICAS}

Bahloul, J. (2002). Lecturas precarias: Estudio sociológico sobre los “pocos lectores”. Ciudad de México: Fondo de Cultura Económica.

Carlino, P. (2003). Leer textos científicos y académicos en la educación superior: Obstáculos y bienvenidas a una cultura nueva. $6^{\circ}$ Congreso Internacional de la Promoción de la Lectura y el Libro. 
Estudios Pedagógicos XLI, N 1: 143-166, 2015

LAS TEORÍAS IMPLÍCITAS SOBRE LA COMPRENSIÓN TEXTUAL Y LAS ESTRATEGIAS METACOGNITIVAS DE

ESTUDIANTES UNIVERSITARIOS DE PRIMER AÑO

Buenos Aires, Argentina.

Castro, A. (2006). Teorías implícitas del liderazgo, contexto y capacidad de conducción. Anales de Psicología, vol.22 (1), 89-97.

Dienes, Z. \& Perner, J. (1999). A Theory of Implicit and Explicit Knowledge. Behavioral and Brain Sciences, (22), 735-808.

Estrada, C., Oyarzún, M. e Yzerbit, V. (2007). Teorías implícitas y esencialismo psicológico: Herramientas conceptuales para el estudio de la relaciones entre y dentro de los grupos. Psikhe, vol.16 (1), 111-121.

Fodor, J. (1995). Las actitudes proposicionales y el lenguaje del pensamiento. En E. Rabossi (Comp.), Filosofía de la mente y ciencia cognitiva (pp. 174-203). Barcelona: Paidós.

Gómez, V. y Guerra, P. (2012). Teorías implícitas respecto a la enseñanza y el aprendizaje: ¿Existen diferencias entre profesores en ejercicio y estudiantes de pedagogía? Estudios Pedagógicos, vol.38 (1), 25-43.

González, K. (2008). Propuesta de un programa para mejorar la comprensión de textos en estudiantes universitarios. Revista Actualidades Investigativas en Educación, vol.8 (2), 1-31.

Hernández, G. (2008). Teorías implícitas de lectura y conocimiento metatextual. Revista Mexicana de Investigación Educativa, vol.13 (38), 737-771.

Jiménez, A. (2002). Nivel educativo y teorías implícitas sobre la enseñanza: Estructura de creencias en los docentes de educación infantil y primaria, secundaria y superior. Tesis Doctoral. Santiago: Pontificia Universidad Católica de Chile.

Karmiloff-Smith, A. (1997). Auto-organización y cambio cognitivo. Substratum, vol.1 (1), 19-43. (1984). Children's Problem Solving. In M. E. Lamb, A. L. Brown \& B. Rogoff (Eds.), Advances in Developmental Psychology. Vol. 3. (pp.39-90). Hillsdale, N. J.: Lawrence Erlbaum Associates.

Makuc, M. (2013). Teorías implícitas de los profesores acerca de la comprensión de textos. Punta Arenas: Universidad de Magallanes.

(2012). La comprensión textual en el discurso de estudiantes universitarios: Aproximación desde el significado ideacional manifestado en la cláusula. CoDiS Working Papers, (1), 23-40.

(2011). Teorías implícitas sobre comprensión textual y la competencia lectora de estudiantes de primer año de la Universidad de Magallanes. Estudios Pedagógicos, vol.37 (1), 236-253. (2009). Estudio sobre la comprensión de textos en estudiantes de primer año de la Universidad de Magallanes. Revista Centro de Estudios Australes, (4), 109-118.

(2008). Teorías implícitas de los profesores acerca de la comprensión de textos. Revista Signos. Estudios de Lingüística, vol.41 (68), 403-422.

Marrero, J. (2009). Escenarios, saberes y teorías implícitas del profesorado. En J. Marrero (Ed.), El pensamiento reencontrado (pp. 8-44). Barcelona: Octaedro.

(1988). Las teorías implícitas y la planificación del profesor. Tesis Doctoral. Tenerife: Universidad de la Laguna.

Martínez, J. (2009). Las teorías implícitas como conocimiento práctico del profesorado en formación inicial. En J. Marrero (Ed.), El pensamiento reencontrado (pp. 208-217). Barcelona: Octaedro.

More, P. y Narciso, E. (2011). Modelos epistémicos de la lectura en estudiantes mexicanos. Revista Mexicana de Investigación Educativa, vol.16 (51), 1197-1225.

Paris, S., Lipson, M. \& Wixson, K. (1994). Becoming a Strategic Reader. In H. Singer \& B. Rudell (Eds.), Theoretical Models and Processes of Reading (pp.778-862). Newark, Delaware: IRA.

Parodi, G. (2009). El corpus académico y profesional del español PUCV 2006: Semejanzas y diferencias entre los géneros académicos y profesionales. Estudios Filológicos, (44), 123-147.

(2007). Comprensión y aprendizaje a partir del discurso especializado escrito: Teoría y empiria. En G. Parodi (Ed.), Lingüística de corpus y discursos especializados: Puntos de mira (pp. 223-258). Valparaíso: Ediciones Universitarias de Valparaíso. 
(2005). La comprensión del discurso especializado escrito en ámbitos técnicoprofesionales: ¿Aprendiendo a partir del texto? Revista Signos. Estudios de Lingüística, vol.38 (58), 221-267.

Peredo, M. (2003). La identidad lectora y los pensamientos que se activan durante la lectura de los textos de auto ayuda. $6^{\circ}$ Congreso Internacional de la Promoción de la Lectura y el Libro. Buenos Aires, Argentina.

Peronard, M. (2005). La metacognición como herramienta didáctica. Revista Signos. Estudios de Lingüística, vol.38 (57), 61-74.

., Velásquez, M., Crespo, N. y Viramonte, M. (2002). Conocimiento metacognitivo del lenguaje escrito: Instrumento de medida y fundamentación teórica. Infancia y Aprendizaje, vol.25 (2), 131-145.

., Crespo, N. y Velásquez, M. (2000). La evaluación del conocimiento metacomprensivo en alumnos de educación básica. Revista Signos. Estudios de Lingüística, vol.33 (47), 167-180.

Pozo, J. I., Scheuer, N., Mateos, M. y Pérez Echeverría, M. (2006). Las teorías implícitas sobre el aprendizaje y la enseñanza. En J. I. Pozo, N. Scheuer, M. Pérez Echeverría, M. Mateos, E. Martín y M. de la Cruz (Eds.), Nuevas formas de pensar la enseñanza y el aprendizaje. Las concepciones de profesores y alumnos (pp. 95-127). Barcelona: Graò.

. (2003). Adquisición del conocimiento. Madrid: Morata.

(2001). Humana mente. El mundo, la conciencia y la carne. Madrid: Morata.

y Rodrigo, M. (2001). Del cambio de contenido al cambio representacional en el conocimiento conceptual. Infancia y Aprendizaje, vol.24 (4), 407-423.

., Pérez, M. P., Sanz, A. y Limón, M. (1992). Las ideas de los alumnos sobre la ciencia como teorías implícitas. Infancia y Aprendizaje, (57), 3-21.

y Carretero, M. (1987). Del pensamiento formal a las concepciones espontáneas: ¿Qué cambia en la enseñanza de la ciencia? Infancia y Aprendizaje, (38), 35-42.

Rivera, M. J. (2000). Estrategias de lectura para la comprensión de textos escritos: El pensamiento reflexivo y no lineal en alumnos de educación superior. Revista Digital Umbral 2000, (12), 1-6.

Rodrigo, M. J. y Correa, N. (2001). Representación y procesos cognitivos: Esquemas y modelos mentales. En C. Coll, J. Palacios y A. Marchesi (Comps.), Desarrollo psicológico y educación, 2. Psicología de la educación escolar (pp. 117-135). Madrid: Alianza.

. (1997). Del escenario sociocultural al constructivismo episódico: Un viaje al conocimiento escolar de la mano de las teorías implícitas. En M. J. Rodrigo y J. Arnay (Comps.), La construcción del conocimiento escolar (pp. 177-191). Barcelona: Paidós.

. (1994a). El hombre de la calle, el científico y el alumno: ¿Un solo constructivismo o tres? Investigación en la Escuela, (23), 7-16.

. (1994b). Etapas, contextos, dominios y teorías implícitas en el conocimiento social. En M. J. Rodrigo (Ed.), Contexto y desarrollo social (pp. 26-46). Madrid: Síntesis.

(1993). Representaciones y procesos en las teorías implícitas. En M. J. Rodrigo, A. Rodríguez y J. Marrero (Eds.), Las teorías implícitas. Una aproximación al conocimiento cotidiano (pp. 95-117). Madrid: Visor.

., Rodríguez, A. y Marrero, J. (1993). Las teorías implícitas. Una aproximación al conocimiento cotidiano. Madrid: Visor. $145-156$.

(1985). Las teorías implícitas en el conocimiento social. Infancia y Aprendizaje, (31-32),

Rodríguez, E. (2001). Teoría implícita y formación inicial del profesorado de Educación Media. Revista Enfoques Educacionales, vol.3 (2), 145-155.

Ruiz, M. (2009). Evaluación de lengua escrita y dependencia de lo literal. Barcelona: Graò.

Schraw, G. (2000). Reader Beliefs and Meaning Construction in Narrative Text. Journal of Educational Psychology, vol.92 (1), 96-106.

\& Bruning, R. (1996). Readers' Implicit Models of Reading. Reading Research 
Estudios Pedagógicos XLI, N 1: 143-166, 2015

LAS TEORÍAS IMPLÍCITAS SOBRE LA COMPRENSIÓN TEXTUAL Y LAS ESTRATEGIAS METACOGNITIVAS DE ESTUDIANTES UNIVERSITARIOS DE PRIMER AÑO

Quarterly, vol.31 (3), 290-305.

Velásquez, M., Cornejo, C. y Roco, A. (2008). Evaluación de la competencia lectora en estudiantes de primer año de carreras del área humanista y carreras del área de la salud en tres universidades del Consejo de Rectores. Estudios Pedagógicos, vol.33 (1), 123-138.

Vélez, G. (2006). Las autobiografías lectoras como autobiografía de aprendizaje. En J. I. Pozo, N. Scheuer, M. Pérez Echeverría, M. Mateos, E. Martín y M. de la Cruz (Eds.), Nuevas formas de pensar la enseñanza y el aprendizaje. Las concepciones de profesores y alumnos (pp. 307-319). Barcelona: Graò. 